\title{
Correlation between obstetric outcome and amniotic fluid index (AFI) in preterm prelabour rupture of membranes (PPROM)
}

\author{
Anusree Saraswathy ${ }^{1}$, Jayshree V. Vaman ${ }^{2 *}$, Mayadevi Brahmanandan², Nirmala C. ${ }^{2}$
}

\author{
${ }^{1}$ Department of Obstetrics and Gynecology, Aster Hospital, Aster DM Healthcare, Dubai, UAE \\ ${ }^{2}$ Department of Obstetrics and Gynaecology, SATH, Government Medical College, Thiruvananthapuram, Kerala, \\ India
}

Received: 10 November 2018

Accepted: 23 November 2018

\author{
*Correspondence: \\ Dr. Jayshree V. Vaman, \\ E-mail: jysree@gmail.com
}

Copyright: (c) the author(s), publisher and licensee Medip Academy. This is an open-access article distributed under the terms of the Creative Commons Attribution Non-Commercial License, which permits unrestricted non-commercial use, distribution, and reproduction in any medium, provided the original work is properly cited.

\begin{abstract}
Background: The purpose was to determine whether AFI $<5 \mathrm{~cm}$ after preterm premature rupture of the membranes (PPROM) is associated with an increased risk of maternal and perinatal morbidity.

Methods: We performed a prospective case control study of 161 singleton pregnancies complicated by preterm prelabour rupture of the membranes (PPROM) in whom AFI was assessed. Patients were categorized in two groups on the basis of amniotic fluid index- AFI $<5 \mathrm{~cm}$ or AFI $\geq 5 \mathrm{~cm}$. Categorical data were tested for significance with the $\chi 2$ and Fisher exact tests. All 2 -sided $\mathrm{p}$ values $<0.05$ were considered significant.

Results: Both groups were similar with respect to selected demographics, gestational age at rupture of the membranes, gestational age at the delivery, birth weight. Both groups were similar with respect to maternal chorio-amnionitis, abruption, mode of delivery, early onset neonatal sepsis and NICU stay. Patients with $\mathrm{AFI}<5 \mathrm{~cm}$ demonstrated greater frequency of $\mathrm{C} / \mathrm{S}$ delivery for non-reassuring fetal tests.

Conclusions: There is no significant difference between obstetric outcome in AFI $<5$ and AFI 5 after PPROM between 24- and 37-weeks' gestation.
\end{abstract}

Keywords: Amniotic fluid index (AFI), Obstetric outcome, Preterm prelabour rupture of membranes (PPROM)

\section{INTRODUCTION}

Prelabour rupture of membrane constitute one of the most important dilemmas in current obstetric practice. This term is applied to leakage of amniotic fluid in the absence of labour, irrespective of gestational age. Prelabour membrane rupture before 37 weeks of gestation is referred to as preterm prelabour rupture of membranes (PPROM). Overall, about $10 \%$ of all gestation are complicated by prelabour membrane rupture. Incidence of PPROM is about $3 \%$ of all pregnancies. The consequences of PPROM for the neonate fall into three major overlapping categories. The first is the significant neonatal morbidity and mortality associated with prematurity. Second are the complications during labour and delivery that increase the risk for neonatal resuscitation and thirdly infection. Maternal complication includes infection and increased risk of caesarean section. A lot of research has gone into cases of PPROM to ascertain the optimal timing and route of delivery so as to minimise maternal and perinatal morbidity. Several key parameters have been devised to aid in these decisions. Ultrasound has emerged as a useful tool in detecting and predicting prognostic factors. Amniotic fluid index especially low AFI has been used as a predictor for adverse outcome by many consultants and several studies support its use as a tool to decide on optimal mode of delivery. Also, in making decisions regarding referral to a higher centre with better neonatal facilities. 
It has also been proposed that amniotic fluid posse's certain bacteriostatic properties that protect against potential infections processes and that a decrease in amniotic fluid volume may impair the gravid women ability to combat such infections. Vintziuleos et al. ${ }^{1}$ These investigations demonstrated that patients with oligo-hydramnios $(\mathrm{AFI}<5)$, were at greater risk of having chorioamnionitis and subsequent sepsis in the neonate. ${ }^{1,2}$ The purpose of present study is to determine whether patients with PPROM and amniotic fluid index $<5 \mathrm{~cm}$ are at an increased risk of having perinatal morbidity.

\section{METHODS}

The purpose was to determine whether $\mathrm{AFI}<5 \mathrm{~cm}$ after preterm prelabour rupture of the membranes (PPROM) is associated with an increased risk of maternal and perinatal morbidity when compared to patients with PPROM and AFI $>5$. Prospective observational case control study. The study is hospital based, setting is Sri Avittam Thirunanl (SAT) Hospital, Medical college, Thiruvananthapuram. Sample size calculation was based on relevant statistical formula and fixed at 161 .

\section{Inclusion criteria}

- Singleton pregnancies complicated by preterm premature rupture of membranes, between gestational age 24 weeks to 37 weeks that admitted in the labour room of SAT hospital during the period of study.

- Those cases who could get a scan done and AFI determined prior to delivery.

\section{Exclusion criteria}

- All cases of PPROM with multiple pregnancy, diabetes, severe pre-eclampsia complicating pregnancy, IUD, anomalies.

- Those cases of PPROM, whose AFI could not be determined prior to delivery.

Authors performed a prospective case control study of 161 singleton pregnancies complicated by preterm prelabour rupture of the membranes (PPROM) with delivery between 24- and 37-weeks gestation. In all eligible women, ultrasound was performed for the evaluation of amniotic fluid index. Patients were categorized to two groups on the basis of amniotic fluid index $<5$ or AFI $\geq 5 \mathrm{~cm}$. These women were followed and monitored up to delivery. The women of the two groups did not have significant difference in age, gestational age at the time of ruptured membrane, body mass index, gravidity, parity and gestational age at delivery. The routine investigations were done in all the cases. In labour, antibiotics were started, and she was watched for progress, fetal distress, blood stained liquor. Mode of delivery was noted. After delivery, a detailed neonatal examination was done by the paediatrician to determine the APGAR score, birth weight, birth asphyxia respiratory distress and evidence of sepsis. If necessary, neonatal ICU admission was sought for the preterm neonate. All stillbirths and neonatal deaths were noted. Postnatally, the patients were observed for postpartum pyrexia, foul smelling lochia and antibiotics were continued. Data were tested for significance with the $\chi^{2}$, Fisher exact tests and for significance with the student $t$ test. All $\mathrm{p}$ values $<0.05$ were considered significant.

\section{RESULTS}

Majority of cases with rupture of membranes occurring after 34 weeks were either induced or delivered spontaneously before an USS could be done. As a result, Amniotic Fluid Index (AFI) could be obtained only for 161 cases. The study group comprised 161 cases of PPROM after satisfying the exclusion criteria. AFI $<5$ was noted in 19 patients and AFI > 5 was noted in 142 cases (Figure 1).

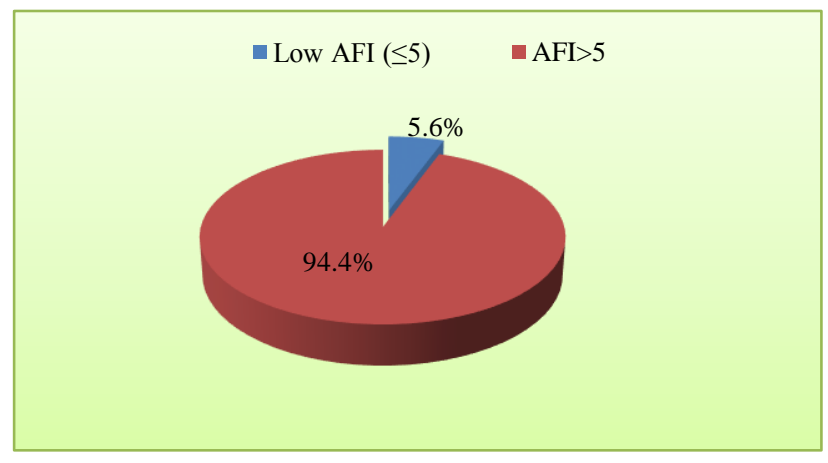

Figure 1: Liquor volume

The majority of patients in this study are in the age group 20-24 years. The mean age of the <5 AFI group was 23.2 years. $77.5 \%$ of cases are primi gravidas. The predominance of primi gravidas in the PPROM sample is as anticipated. The mean gestational age at PPROM in the <5 AFI group was 33.2 weeks. In $52.9 \%$ of cases, membrane rupture occurred between 28-34 weeks, in $44.2 \%$ it occurred between 34-37 weeks and in $3 \%$ between 24-28 weeks. Majority of the babies (92.9\%) were low birth weight babies. Mean birth weight of both the AFI groups were comparable (Table 1).

Table 1: Comparing the obstetric outcome between AFI $<5$ and AFI $>5$.

\begin{tabular}{|lll|}
\hline Parameter & AFI $<5$ & AFI $>5$ \\
\hline Mean maternal age & 23.2 years & $24.5 y e a r s$ \\
\hline Mean gestational age & 33.2 weeks & 33.9 weeks \\
\hline Mean birth weight & $1.8 \mathrm{~kg}$ & $1.93 \mathrm{~kg}$ \\
\hline Mean NICU stay days & 38 days & 28 days \\
\hline
\end{tabular}

The obstetric outcome measures studied were maternal chorioamnionitis, maternal abruption, mode of delivery, New born sepsis and NICU stay (Table 2). Chorioamnionitis was present in only 2 patients with AFI 
$<5$ compared to 27 patients in AFI > 5, but the difference is not statistically significant.

No cases of abruption was reported in $\mathrm{AFI}<5$ group, whereas 4 cases were reported in AFI $>5$ group. The finding is not significant statistically. $44.44 \%$ with AFI $<5$ needed neonatal ICU stay. In the group with AFI $>5$, a similar percentage was noted.
No association was obtained. Newborn sepsis and mode of delivery were comparable between the two groups. 5 out of 19 cases of AFI $<5$ ended up in caesarean section and in 3 out of the 5 cases the indication for caesarean section was fetal distress. In the AFI > 5 group, failed induction was the reason for CS in $17.1 \%$, non-vertex presentation in $22.85 \%$, abruption in $5,7 \%$ and previous CS in $25.7 \%$ (Table 3 ).

Table 2 Comparing the adverse outcome between AFI $<5$ and AFI $>5$.

\begin{tabular}{|c|c|c|c|c|}
\hline Parameter & & AFI <5 No. $(\%)$ & AFI >5 No. (\%) & P value \\
\hline \multirow{2}{*}{ Chorioamnionitis } & Present & $2(0.105)$ & $27(0.167)$ & \multirow{2}{*}{$>0.05$} \\
\hline & Absent & 17 & 115 & \\
\hline \multirow{2}{*}{ Abruption } & Present & 0 & $4(0.024)$ & \multirow{2}{*}{$>0.05$} \\
\hline & Absent & 19 & 138 & \\
\hline \multirow{2}{*}{ NICU stay } & Present & $14(0.736)$ & $65(0.403)$ & \multirow{2}{*}{$>0.05$} \\
\hline & Absent & 5 & 77 & \\
\hline \multirow{2}{*}{ Newborn sepsis } & Present & $3(0.157)$ & $52(0.322)$ & \multirow{2}{*}{$>0.05$} \\
\hline & Absent & 16 & 90 & \\
\hline \multirow{2}{*}{ Mode of delivery } & LSCS & $5(0.263)$ & $25(0.155)$ & \multirow{2}{*}{$>0.05$} \\
\hline & Vaginal delivery & 14 & 117 & \\
\hline
\end{tabular}

Table 3: Liquor volume and indication for LSCS.

\begin{tabular}{|lllllll|} 
Liquor volume & Failed induction & Breech PPROM & Abruption & Fetal distress & Transverse lie Previous CS \\
\hline AFI $\leq 5$ & $2(40 \%)$ & - & - & $3(60 \%)$ & - & - \\
\hline AFI $>5$ & $6(17.1 \%)$ & $7(20 \%)$ & $2(5.7 \%)$ & $2(5.7 \%)$ & $1(2.85 \%)$ & $7(25.7 \%)$ \\
\hline
\end{tabular}

\section{DISCUSSION}

Huang et al in their study show an association between oligohydramnios and adverse maternal and neonatal outcome in PPROM. ${ }^{3}$ No such association was found in present study possibly because number of patients with low AFI in this study is not high enough to rule out possible association of oligohydramnios with adverse maternal and perinatal outcome. The impact of low amniotic fluid volume on maternal and fetal outcomes were analysed. Caesarean section rate in this study was $12.1 \%$ which is similar to that reported in the study from Punjab (Tahir S et al). ${ }^{4}$ It is low compared to the study by Charles P J et al5 in which the incidence was $58 \% .^{5}$ Nonvertex presentations (breech and transverse lie) accounted for $22.85 \%$ of cases in present study.

$10 \%$ of caesarean section were due to fetal distress. A study by Borna et al showed that oligohydramnios associated with a higher likelihood of caesarean section due to non-reassuring fetal heart rate patterns. ${ }^{6}$ This was consistent with other studies as well. Failed induction accounted for $26.6 \%$ of caesarean sections, $30 \%$ were previous sections admitted with PPROM. The incidence of failed induction and operative morbidity is higher at earlier gestational ages (Julien SK and Olasewere T). ${ }^{7}$
According to Huang S. et al, group of oligohydramnios had high rates of caesarean section, chorioamnionitis, fetal distress, neonatal asphyxia, early-onset neonatal sepsis than those in the group of normal amniotic fluid volume and that oligohydramnios was an independent risk factor of chorioamnionitis and early-onset neonatal sepsis. ${ }^{3}$ A similar association of AFI scores less than 5 with chorioamnionitis and early onset neonatal sepsis was identified by Borna et al in 2004 These studies were designed with a primary objective of assessing low AFI as a risk factor for adverse neonatal outcome and for chorioamnionitis. $^{6}$ However no such association was found present study possibly because number of patients with low AFI in this study is not high enough to rule out possible association of oligohydramnios with adverse maternal and fetal outcome. Inter observer variations can be significant in ultrasound assessment of liquor volume. This might have contributed to disparity with observations from other studies. Fetal distress predominated among the indications for caesarean section in the group with low AFI.

Mean length of NICU stay in $<5$ AFI group in present study is 38 days compared to 28 days in the $>5$ AFI group which is comparable to the study by Ladella et al Length of stay in NICU was 42 days with AFI less than 5 
$\mathrm{cm}$ versus 26.5 days with AFI greater than $5 \mathrm{~cm}(\mathrm{P}<$ 0.013). ${ }^{8}$ Unlike the study by Piazze et al, we could find no significant association between AFI and APGAR scores, neonatal RDS and maternal chorio-amnionitis. ${ }^{9}$ Mosavi et al in their study found an association between AFI $<5$ and neonatal sepsis and neonatal death. ${ }^{10}$ They suggested that amniotic fluid index $<5 \mathrm{~cm}$ should be considered as a warning sign for predicting poor prognosis of pregnancy complicated by preterm premature rupture of membranes. However, no such correlation were arrived at by the present study.

Funding: No funding sources

Conflict of interest: None declared

Ethical approval: The study was approved by the Institutional Ethics Committee

\section{REFERENCES}

1. Vintzileos AM, Cmpbell WA, Nochimson DJ: degree oligohydramnios and pregnancy outcome in patients with prom. Obstet Gynecol. 1985,66(2):1627.

2. Vermillion S, Kooba A: Amniotic fluid index value after prom and subsequent perinatal infection. Am J Obstet Gynecol. 2000;183(2):271-6.

3. Huang S, Qi HB, Li L. Residue amniotic fluid volume after preterm premature rupture of membranes and maternal-fetal outcome. Zhonghua fu chan ke za zhi. 2009;44(10):726-30

4. Tahir S, Aleem M, Aziz R. Incidence And outcome of Preterm premature rupture of membranes. Pak J Med Sci. 2002;18(1):26-32

5. Charles PJ, Muriel R, Charles PJ, Rene E, Oliver C, Pascal G, et al. A prospective population-based study of 598 cases of PPROM between 24- and 34-weeks gestation: description, management and mortality (Dominos cohort). Eur J Obstet Gynec. 2005;121(2):164-70.

6. Borna S, Borna H, Hantoushzadeh S. 'Perinatal outcome in preterm premature rupture of membranes with Amniotic fluid index $<5(\mathrm{AFI}<5)$. BMC Preg Childbirth. 2004;4(1):15.

7. Julien S, Khandelwal M, Olasewere T. randomized Trial Comparing Long-term Versus Short-term Antibiotic Prophylaxis In Preterm Premature Rupture Of Membranes (pprom).: 31. Am J Obstet Gynecol. 2002;187(6):S66.

8. Ladella S, Leung T, Cortez C. Effects of Amniotic Fluid Index on Perinatal Outcomes in Preterm Premature Rupture of Membranes [37o]. Obstet Gynecol. 2017;129:S162.

9. Piazze J, Anceschi MM, Cerekja A, Brunelli R, Meloni P, Marzano S, Cosmi E: Validity of amniotic fluid index in preterm rupture of membranes. J Perinat Med. 2007;35(5):394-8

10. Mousavi AS, Hashemi N, Kashanian M, Sheikhansari N, Bordbar A, Parashi S: Comparison between maternal and neonatal outcome of PPROM in the cases of amniotic fluid index (AFI) of more and less than $5 \mathrm{~cm}$. J Obstet Gynaecol. 2018;38(5):611-5.

Cite this article as: Saraswathy A, Vaman JV, Brahmanandan M, Nirmala C. Correlation between obstetric outcome and amniotic fluid index (AFI) in preterm prelabour rupture of membranes (PPROM). Int J Reprod Contracept Obstet Gynecol 2018;7:4858-61. 